IAC-09.A5.2.-B3.6.7

\title{
ASSESSMENT OF ROBOTIC RECON FOR HUMAN EXPLORATION OF THE MOON
}

\author{
Terrence Fong \\ NASA Ames Research Center, Moffett Field, California, USA \\ terrence.fong@nasa.gov
}

\begin{abstract}
Andrew Abercromby, Wyle Laboratories, Houston, Texas, USA, andrew.f.abercromby@nasa.gov
Maria G. Bualat, NASA Ames Research Center, Moffett Field, California, USA, maria.g.bualat@nasa.gov

Matthew C. Deans, NASA Ames Research Center, Moffett Field, California, USA, matthew.c.deans@nasa.gov

Kip V. Hodges, Arizona State University, Tempe, Arizona, USA, kvhodges@asu.edu

José M. Hurtado, Jr., University of Texas, El Paso, Texas, USA, jhurtado@utep.edu

Rob Landis, NASA Ames Research Center, Moffett Field, California, USA, rob.r.landis@nasa.gov

Pascal Lee, Mars Institute, Moffett Field, California, USA, pascal.lee@marsinstitute.net

Debra Schreckenghost, TRACLabs, Inc., Houston, Texas, USA, schreck@traclabs.com
\end{abstract}

\begin{abstract}
Robotic reconnaissance ("recon") has the potential to significantly improve scientific and technical return from lunar surface exploration. In particular, robotic recon can be used to improve traverse planning, reduce operational risk, and increase crew productivity. To study how robotic recon can benefit human exploration, we recently conducted a field experiment at Black Point Lava Flow (BPLF), Arizona. In our experiment, a simulated ground control team at NASA Ames teleoperated a planetary rover to scout geology traverses at BPLF. The recon data was then used to plan revised traverses. Two-man crews subsequently performed both types of traverses using the NASA "Lunar Electric Rover" (LER) and simulated extra-vehicular activity (EVA) suits. This paper describes the design of our experiment, presents our preliminary results and discusses directions for future research.
\end{abstract}

\section{INTRODUCTION}

$\mathrm{T}$ he planned human return to the Moon offers new opportunities to advance the scientific exploration of the lunar surface. However, when the new exploration campaign begins, short human missions (lasting a few weeks) will be separated by several months, during which time robots could perform work [1]. A central challenge, therefore, is to coordinate human and robotic activities to maximize scientific return. We believe that one way to do this is with robotic recon.

We define robotic recon as "remotely operating a planetary rover to scout planned sorties prior to EVA". Scouting is an essential phase of field work, particularly for geology. Robot instruments can provide observations of the surface and subsurface geology at resolutions and from viewpoints not achievable from orbit. This surface-level data can then be used to improve planning and crew performance.

Since 2008, we have been developing and evaluating systems, operational concepts, and protocols for robotic recon $[2,3]$. Our approach is inspired by the Mars Exploration Rovers (MER), as well as human spaceflight, including Apollo, the Space Shuttle, and the International Space Station (ISS). Our ground con- trol, for example, integrates a science team based on both the MER Science Operations Working Group (SOWG) [4] and the Apollo "Science Backroom" [5].

We hypothesize that robotic recon improves human exploration in three ways: (1) it increases scientific understanding so that better traverse plans can be produced; (2) it reduces operational risk by evaluating routes and terrain hazards; and (3) it improves crew productivity by facilitating situational awareness. To test these hypotheses, we conducted a field experiment of robotic recon at Black Point Lava Flow (BPLF), Arizona during Summer 2009.

In our experiment, we employed a crossover design in which field geology traverses were planned and executed with, and without, robotic recon data. Initially, two "pre-recon" traverse routes were planned using only orbital images. We then remotely operated a planetary rover equipped with cameras and 3D lidar to scout the traverses. The recon data were subsequently used to develop "post-recon" traverse plans. Finally, the four traverses (pre- and post- recon in two different areas) were executed by two-man crews using the NASA "Lunar Electric Rover" (LER) and simulated EVA suits. Throughout the experiment, we used numerous metrics to assess the impact of robotic recon. 


\section{EXPERIMENTAL DESIGN}

We designed our experiment to study: (1) to what extent robotic recon can reduce uncertainty and improve science merit of traverse planning prior to human fieldwork and (2) how scouting in advance of human missions can improve crew efficiency and the quality of data collection. The experiment involved four phases of activity, which simulated multiple phases of a lunar exploration campaign.

Initial Traverse Planning. During the initial phase, a science team developed "pre-recon" crew traverse plans for geologic exploration of the BPLF site using only orbital imagery and general knowledge of the site. The team then identified high-priority areas where surface-level observations would help reduce planning uncertainties. This information was then used to develop robotic recon traverses.

Robotic Recon Mission Simulation. In the second phase, we teleoperated a planetary rover to perform robotic recon traverses at the site. A simulated ground control team (including science operations, robotics engineering, and flight control) remotely operated the robot from NASA Ames. The mission simulation included an operational timeline inspired by the MER SOWG and a hybrid operations protocol derived from MER and human spaceflight missions.

Post-Recon Planning. After the robotic recon mission was complete, the science team created "postrecon" crew traverse plans by modifying the "prerecon" crew traverses using the data gathered by the robot. During this phase, details about the site that were only possible to observe on the surface were factored into the traverse replanning.

Crew Mission Simulation. The final phase involved execution of the "pre-recon" and "post-recon" traverses by crews using the LER and simulated EVA suits. Two crews each performed one traverse with the benefit of recon information and one traverse without. A "science backroom" remotely supported the crews.

\section{Definitions}

EVA Station. A location where crew performs an EVA.

Recon Station. A location where a robotic rover deploys instruments, collects data, or leaves a marker.

Science Target. A location of scientific interest. A science target may be a point feature (e.g. a boulder), a linear feature (e.g., escarpment), or a bounded area.

Candidate Science Target. A science target that may be added to a traverse plan.

Recon Target. A science target or candidate science target that is selected for recon.

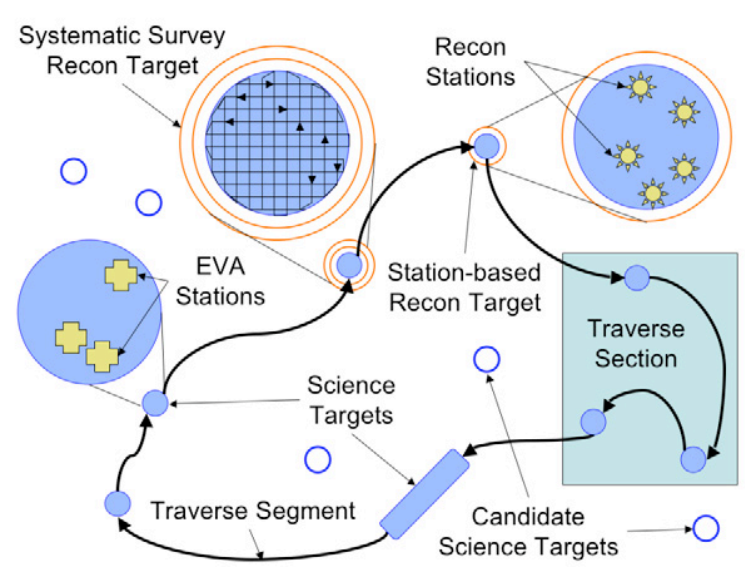

Figure 1. Traverse definitions.

Station-based Recon Target. A recon target where the robot collects data at one (or more) recon stations.

Systematic Survey Recon Target. A recon target where the robot is used to collect dense survey measurements.

Traverse Segment. A translation portion of a traverse.

Traverse Section. A portion of a traverse that may include multiple targets and segments.

\section{$\underline{\text { Assumptions }}$}

- The pre-recon traverse plans were developed using only lunar-relevant remote sensing data and limited a priori knowledge of the site.

- Science team members had not previously performed field work at the site.

- Previous missions to the region had collected samples, but had not yet been analyzed.

\section{Ground rules}

- Orbital imagery used for planning did not exceed Lunar Reconnaissance Orbiter (LRO) resolution.

- The science team did not use knowledge of what was seen by crew and science backrooms during previous missions, including prior surface-level data.

- Only data available before recon, and collected during recon, were used for planning and revising the traverses used in this experiment.

- The same science team created both the pre- and post- recon traverse plans.

- Serendipitous discoveries were not considered when assessing metrics.

- Recon for any given target were constrained to reflect a ratio of 14-day crew mission to 6-month robotic mission. 


\section{Black Point Lava Flow}

Black Point Lava Flow (BPLF) is located $65 \mathrm{~km}$ north of Flagstaff, Arizona. It was selected by the NASA Desert Research and Technology Studies (DRATS) project as a lunar analog test site for its geologically relevant features, including outcrops of basaltic volcanic rocks and unit contacts. The size of the test area $\left(\sim 3,000 \mathrm{~km}^{2}\right)$ and abundance of geologic features enables extended range simulated science sorties [6].

\section{Science Objectives}

To facilitate analog field testing at BPLF, D-RATS has defined a set of notional science objectives for lunar mission simulations. The primary notional objective is: determine the origin, nature, and relative ages of the geologic units to determine the geologic history of the site. Supporting notional objectives are:

- Characterize the BPLF, in particular its age, morphology, structure, petrology, mineralogy, chemistry, and spatial and temporal variations;

- Determine the relationship of BPLF to other lava flows and volcanic features;

- Characterize the other geologic units and their relation to the BPLF in space and time; and

- Determine the geologic history of the site and determine the absolute ages to the major units in so far as possible.

We used these notional objectives as guidelines for traverse planning and execution in our experiment.

\section{EFFECT ON TRAVERSE PLANNING}

We expected that robotic recon can improve traverse planning by helping reduce scientific and operational uncertainties (route selection, trafficability, etc). For example, robotic recon should enable more precise targeting of accessible locations that are likely to yield higher science return. To study this, we developed three hypotheses to evaluate the effect of robotic recon on traverse planning (Table 1).

Table 1. Traverse planning hypotheses.

\begin{tabular}{|c|l|}
\hline$\#$ & \multicolumn{1}{c|}{ Hypothesis } \\
\hline 1A & $\begin{array}{l}\text { Robotic recon can improve the science poten- } \\
\text { tial of a traverse plan. }\end{array}$ \\
\hline 1B & $\begin{array}{l}\text { Robotic recon can substantially change the } \\
\text { design of a traverse plan. }\end{array}$ \\
\hline 1C & $\begin{array}{l}\text { Robotic recon can reduce the science uncer- } \\
\text { tainty in a traverse plan. }\end{array}$ \\
\hline
\end{tabular}

\section{Science Potential (Hypothesis 1A)}

To test the hypothesis that "robotic recon can improve the science potential of a traverse plan", we employ a "science potential" rating scale (Table 2). This metric is a qualitative estimate of how well a target may help address the science objectives for exploration of the BPLF. A target that is believed to facilitate acquiring key observations, samples, etc. will have a better rating than a target that does not.

Table 2. Science potential rating scale.

\begin{tabular}{|c|l|}
\hline Descriptor & \multicolumn{1}{c|}{ Definition } \\
\hline Poor & $\begin{array}{l}\text { Limited potential to address science } \\
\text { objectives }\end{array}$ \\
\hline Fair & $\begin{array}{l}\text { Some potential to confirm existing } \\
\text { hypotheses and facts }\end{array}$ \\
\hline Good & $\begin{array}{l}\text { Good potential to elucidate existing } \\
\text { hypotheses in detail }\end{array}$ \\
\hline Very good & $\begin{array}{l}\text { Likely will help address scientific } \\
\text { objectives or identify new questions }\end{array}$ \\
\hline Excellent & $\begin{array}{l}\text { Significant opportunity to resolve } \\
\text { scientific questions }\end{array}$ \\
\hline
\end{tabular}

To evaluate science potential, we asked the science team to rate science targets on both the pre- and postrecon traverses prior to the crew mission simulation. We also asked geologists to provide ground truth by rating each station. To test the hypothesis, we will compare all the ratings.

\section{Qualitative Change (Hypothesis 1B)}

To test the hypothesis that "robotic recon can substantially change the design of a traverse plan", we employ a "qualitative change" rating scale (Table 3). This metric assesses the extent to which targets change based on recon. The metric considers several factors: location, objectives, activities, and priority.

Table 3. Qualitative change rating scale.

\begin{tabular}{|c|c|c|}
\hline Descriptor & Location & Objectives \\
\hline Insignificant & no change & $\begin{array}{l}\text { little (or no) } \\
\text { change }\end{array}$ \\
\hline Small & up to $10 \mathrm{~m}$ & $\begin{array}{c}\text { changed some } \\
\text { objectives }\end{array}$ \\
\hline Medium & $\begin{array}{c}\text { up to } 50 \mathrm{~m} \\
\text { (half EVA range) }\end{array}$ & $\begin{array}{l}\text { changed half of } \\
\text { the objectives }\end{array}$ \\
\hline Large & $\begin{array}{l}\text { up to } 100 \mathrm{~m} \\
\text { (EVA range) }\end{array}$ & $\begin{array}{c}\text { changed most } \\
\text { objectives }\end{array}$ \\
\hline Complete & more than $100 \mathrm{~m}$ & $\begin{array}{l}\text { changed all } \\
\text { objectives }\end{array}$ \\
\hline
\end{tabular}


Table 3. Qualitative change rating scale (continued).

\begin{tabular}{|c|c|c|}
\hline Descriptor & \multicolumn{1}{|c|}{ Activities } & $\begin{array}{c}\text { Priority } \\
\text { (low) to } 5 \text { (high) }\end{array}$ \\
\hline $\begin{array}{c}\text { Insignifi- } \\
\text { cant }\end{array}$ & $\begin{array}{c}\text { little (or no) } \\
\text { change }\end{array}$ & no change \\
\hline Small & $\begin{array}{c}\text { changed some } \\
\text { activities }\end{array}$ & changed by \pm 1 \\
\hline Medium & $\begin{array}{c}\text { changed half of } \\
\text { activities } \\
\text { changed most } \\
\text { activities }\end{array}$ & changed by \pm 2 \\
\hline Complete & $\begin{array}{c}\text { changed all ac- } \\
\text { tivities }\end{array}$ & changed by \pm 4 \\
\hline
\end{tabular}

To evaluate qualitative change, we asked the science team to assess each factor independently and then combine all the factors to obtain a single, overall rating for each target. Depending on the nature of a particular target, factors may not be equally weighted when combined. Adding a new target, or deleting an existing target, is a complete change.

\section{Uncertainty (Hypothesis 1C)}

To test the hypothesis that "robotic recon can reduce the science uncertainty in a traverse plan", we employ the "certainty" rating scale shown in Table 4.

Table 4. Certainty rating scale.

\begin{tabular}{|c|c|}
\hline Descriptor & Definition \\
\hline Dubious & $\begin{array}{c}<5 \% \text { certain, high possibility for } \\
\text { error, little is known about site }\end{array}$ \\
\hline Unclear & $<25 \%$ certain \\
\hline Toss-up & $50 \%$ certain, could go either way \\
\hline Confident & $>75 \%$ certain \\
\hline Indisputable & $\begin{array}{c}>95 \% \text { certain, little doubt, } \\
\text { low ambiguity }\end{array}$ \\
\hline
\end{tabular}

To evaluate uncertainty, we asked the science team to rate science targets on both the pre- and post- recon traverses prior to the crew mission simulation.

\section{EFFECT ON CREW PRODUCTIVITY}

We expected that robotic recon improves crew productivity by enabling execution of planned field work to be more efficient. In particular, recon should enable tasks to be performed better and with reduced overhead. To study this, we developed two hypotheses to evaluate the effect of robotic recon on crew productivity (Table 5).
Table 5. Crew productivity hypotheses.

\begin{tabular}{|c|l|}
\hline \multicolumn{2}{|c|}{ Hypothesis } \\
\hline 2A & $\begin{array}{l}\text { Robotic recon can improves the productivity } \\
\text { of a traverse. }\end{array}$ \\
\hline 2B & $\begin{array}{l}\text { Robotic recon can improve the efficiency of } \\
\text { performing a traverse. }\end{array}$ \\
\hline
\end{tabular}

\section{Crew Productivity (Hypothesis 2A)}

To test the hypothesis that "robotic recon can improve the productivity of a traverse", we use the "Weighted Sum of Completed Traverse Objectives" (WSCTO) metric [7]. The metric is based on the Pavilion Lakes Research Project "Scales of Science Merit and Data Quality", but is applied to individual targets.

Data quality can be characterized using essentially two types of criteria: (1) quantitative, such as signal-tonoise ratio and statistical significance; and (2) qualitative, such as the value of the data from a scientific impact (discovery or confirmation) standpoint.

In this experiment, we assess WSCTO as:

$$
\mathrm{WSCTO}=\sum \mathrm{VT}(\mathrm{n}) \times \mathrm{DQ}(\mathrm{n})
$$

where $\operatorname{VT}(\mathrm{n})$ is the "Value of Target $n$ " (Table 6) and DQ(n) is the "Quality of data collected at target $n$ " (Table 7).

Table 6. WSCTO value of target scale.

\begin{tabular}{|c|c|}
\hline Descriptor & Definition \\
\hline 1 & Low anticipated importance \\
\hline 2 & Moderate anticipated importance \\
\hline 3 & High anticipated importance \\
\hline
\end{tabular}

Table 7. WSCTO Data Quality Scale.

\begin{tabular}{|c|l|}
\hline $\begin{array}{c}\text { Descriptor } \\
1 \\
\text { No data }\end{array}$ & Definition \\
\hline $\begin{array}{c}2 \\
\text { Limited }\end{array}$ & $\begin{array}{l}\text { Video and navigation did not support } \\
\text { scientific observations. }\end{array}$ \\
\hline 3 & $\begin{array}{l}\text { Quantitative data adequate for general } \\
\text { documentation of findings. Provides } \\
\text { useful context and enables efficient } \\
\text { return. Data is marginally publishable. }\end{array}$ \\
\hline $\begin{array}{c}\text { Adequate } \\
\text { Signifi- } \\
\text { cant }\end{array}$ & $\begin{array}{l}\text { Quantitative data adequate to support } \\
\text { documentation of scientific findings } \\
\text { and yielding publishable results. }\end{array}$ \\
\hline $\begin{array}{c}5 \\
\text { Excep- } \\
\text { tional }\end{array}$ & $\begin{array}{l}\text { High quality video, navigation, and } \\
\text { other quantitative data that supports } \\
\text { and enhances scientific merit. }\end{array}$ \\
\hline \multicolumn{2}{|c}{}
\end{tabular}




\section{Crew Efficiency (Hypothesis 2B)}

To test the hypothesis that "robotic recon can improve the efficiency of performing a traverse", we assess the "percent of time on task" (PTT). PTT is indicative of how much time crew is able work on a task (e.g., field geology) vs. performing non-productive activities. Non-productive activities include crew idle time, navigating and driving between targets, locating specific features of interest, etc.

We expect that surface-level data acquired by robotic recon improves the crew's preparedness and facilitates their situational awareness. Consequently, the PTT should be increased for traverses planned with recon data.

\section{INITIAL TRAVERSE PLANNING}

Prior to the robotic recon mission, we convened a science team to review the BPLF science objectives, decide on allowable a priori data (satellite imagery, geologic maps, etc.), organize and assign responsibilities within the science team, and develop traverse plans (both crew and robotic recon). We assigned traverse leads (i.e., principal investigators) to two areas of the BPLF, the "West" and "North" zones (Figure 2).

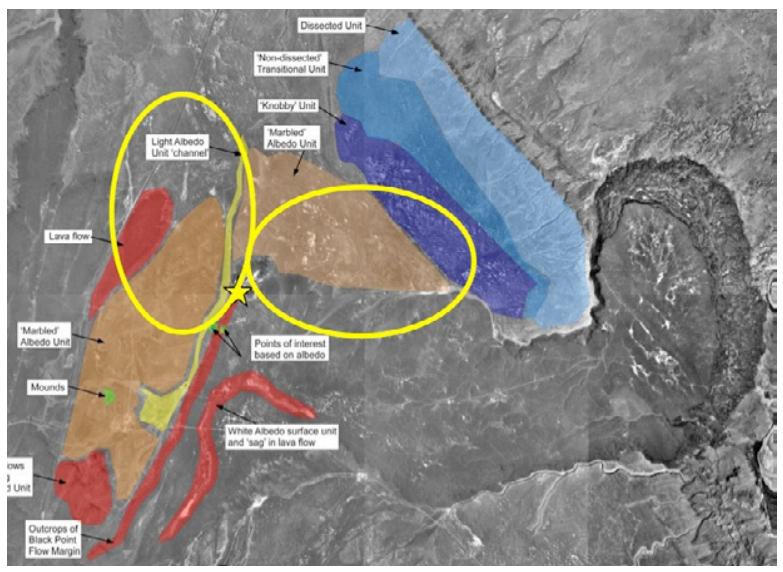

Figure 2. Geologic map of the BPLF. The West and North zones are circled in yellow.

\section{Traverse Planning}

The West and North traverse leads developed two one-day "pre-recon" crew traverses, W1 and N1, using only satellite data and limited knowledge about the site. Satellite data included $60 \mathrm{~cm}$ per pixel, panchromatic QuickBird imagery and 15-90 m per pixel Advanced Spaceborne Thermal Emission and Reflection Radiometer (ASTER) imagery. ASTER provides 14 spectral bands ranging from visible to thermal infrared, which help assess surface composition.
Figure 3 shows the two pre-recon traverses. The pre-recon West traverse, W1, was designed to explore five geologic units in an Apollo-style manner (i.e., rapid area coverage, assumes this is the only time the area will be visited, etc). W1 has 15 stations, covers $10.1 \mathrm{~km}$, and is estimated to require $8: 58 \mathrm{hr}$ to complete (including a total of 6:25 man-hr of EVA).

The pre-recon North traverse, N1, was designed to characterize the northern edge of the BPLF. The traverse is much less exploratory than $\mathrm{W} 1$ and emphasizes sampling the flow edge. $\mathrm{N} 1$ has 6 stations, covers $10.6 \mathrm{~km}$, and is estimated to require $8: 47 \mathrm{hr}$ to complete (including a total of 10:40 man-hr of EVA).

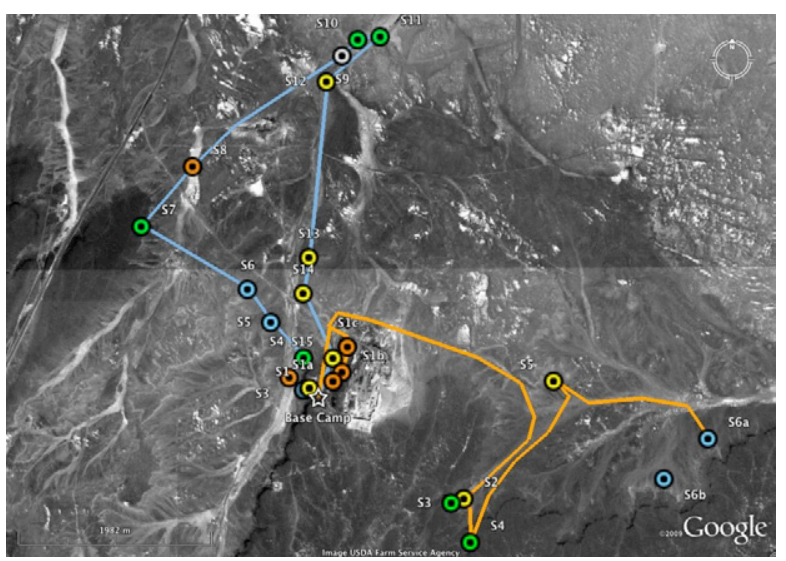

Figure 3. Pre-recon crew traverses, W1 (blue) and $\mathrm{N} 1$ (orange), overlaid on a $60 \mathrm{~cm} /$ pixel base map.

\section{ROBOTIC RECON MISSION SIMULATION}

From 14-27 June 2009, we simulated a lunar robotic recon mission. During this test, we used a NASA Ames "K10" robot (Figure 4) to scout the BPLF. A ground control team remotely operated K10 from the NASA Lunar Science Institute in California.

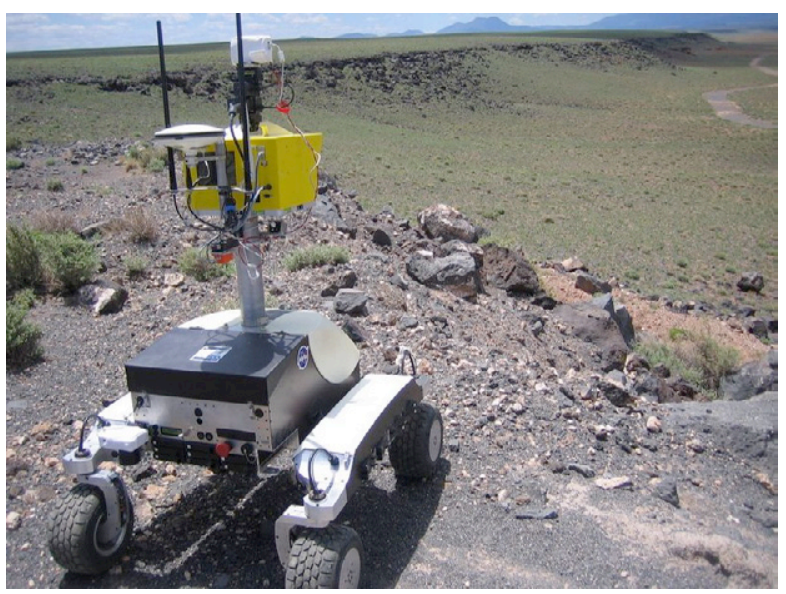

Figure 4. K10 robot operating at BPLF. 
To perform recon, K10 carried three science instruments (Figure 5): an Optech ILRIS-3D scanning lidar (provides 3-D topography measurements); a GigaPan panoramic camera (provides oblique, color images with up to 330 deg field-of-view and gigapixel resolution); and a microscopic imaging camera (provides color images of terrain surface features at 55 microns/pixel).

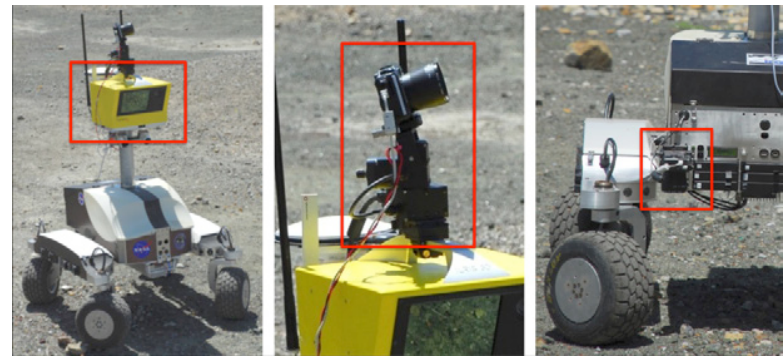

Figure 5. Left to right: (1) 3D scanning lidar, (2) GigaPan camera, (3) microscopic imager.

We remotely operated K10 using a prototype ground control (Figure 6) for lunar surface robotics $[1,8]$. In this ground control, the "Science Operations Team" performs analysis and planning like the MER Science Operations Working Group. The "Flight Control Team" performs real-time, tactical operations similar to human flight missions (Apollo, the Space Shuttle and the International Space Station) [5].

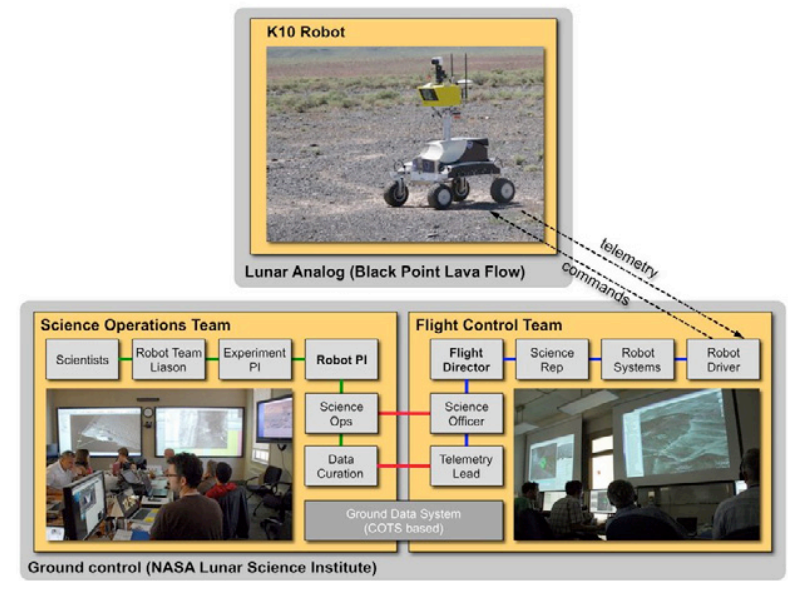

Figure 6. Prototype ground control for lunar surface robotics is a hybrid of Apollo, Space Shuttle, Space Station, and MER operational concepts.

We used "Google Earth" extensively for robot traverse planning and operations [1]. Google Earth is very flexible as a map viewer. It allowed us to display a wide range of geospatial content (image/map overlays, points, etc.) and provided a unified operational view for reviewing site data, robot plans, robot activities, and data collected during recon.

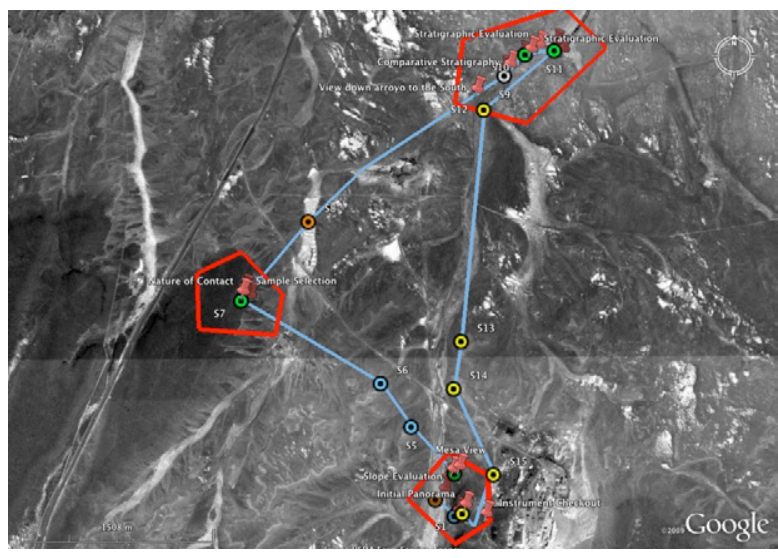

Figure 7. West recon goals. Traverse stations are colored based on priority (green is highest) and three zones (red) have been selected for recon.

Within $\mathrm{W} 1$ and $\mathrm{N} 1$, the traverse leads identified high priority areas where more detailed information was needed to better assess the science merit of targets, or to better assess the accessibility or trafficability of a route or target. Figure 7 shows the recon goals that we developed to scout W1. These focused primarily on reducing the science uncertainty of several targets.

In the North area, the traverse lead identified trafficability of the planned route from station S1c towards the north (Figure 8, orange line) as a concern and requested that robotic recon acquire panoramic imagery to assess the route.

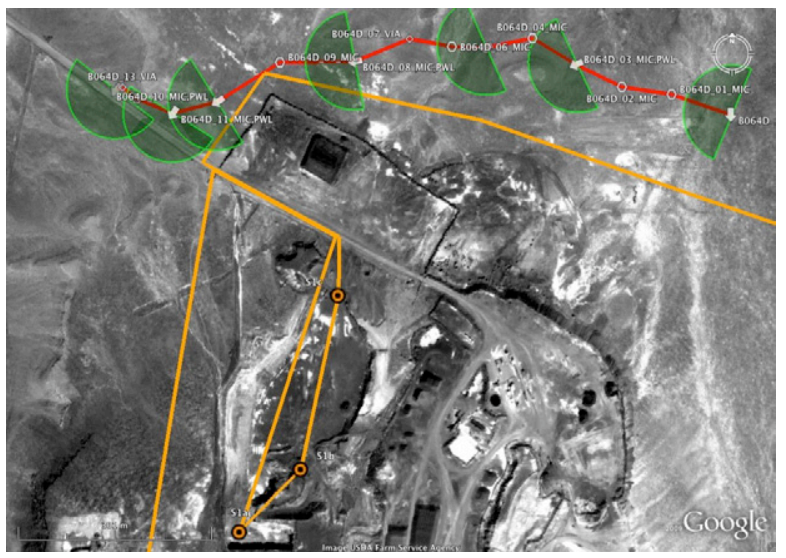

Figure 8. Trafficability of N1 (orange) is a concern north of S1c. Recon (red) addresses this via panoramic images (field-of-view is shown in green).

The science team used Google Earth to develop recon traverse plans by specifying waypoints and data collection activities directly on the map. These traverse plans varied significantly in duration, and complexity. After a plan was defined, the flight control team vetted it (to verify operational constraints) and then executed the plan with the robot. 


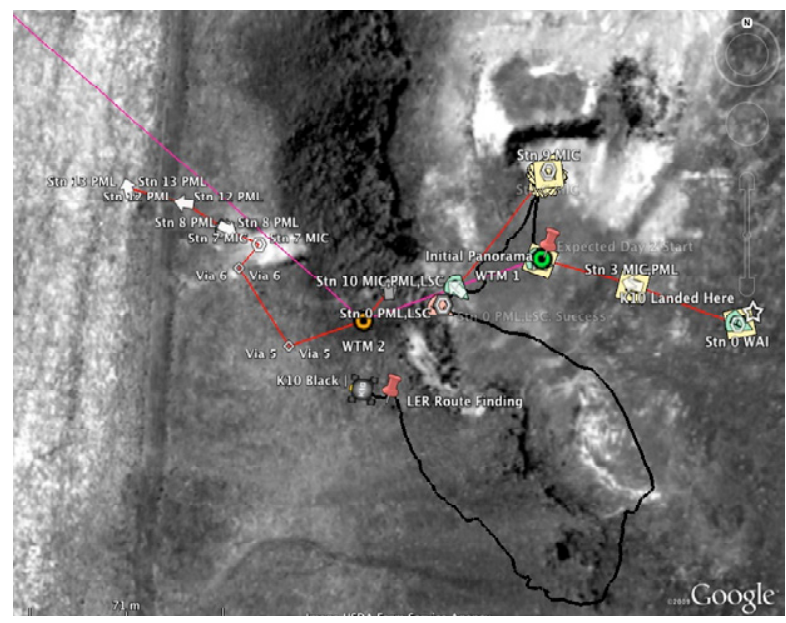

Figure 9. K10 path (black) and geo-located data display (icons) shown in Google Earth.

While K10 was operating, we continuously tracked its location in Google Earth (Figure 9). As K10 acquired recon data, geo-registered placemarks were automatically added to the Google Earth display. Placemarks contained preview images as well as hyperlinks to the K10 "Ground Data System", which allowed scientists to work directly with source data.

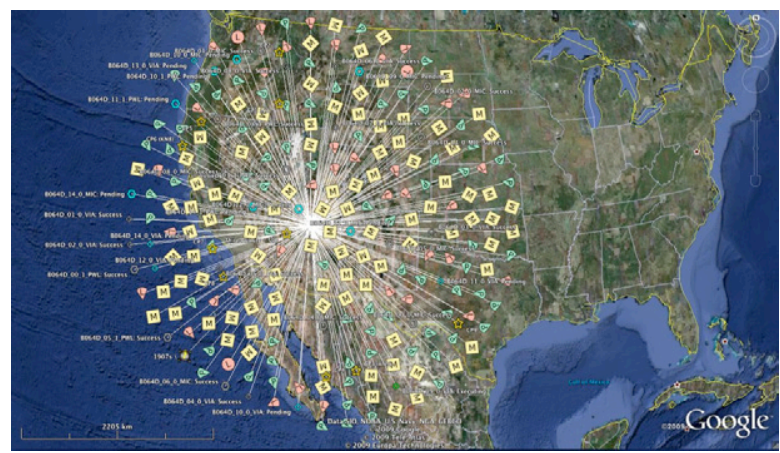

Figure 10. Iconic view of all robotic recon data collected by K10 at BPLF.

By the end of recon operations, K10 had acquired more than $8.5 \mathrm{~GB}$ of data. Figure 10 shows an iconic view of all the recon data: 95 microscopic terrain images are shown as yellow " $\mathrm{M}$ " icons, 39 lidar scans are shown as pink "L" wedges, and 75 GigaPan panoramas are indicated as green "P" wedges.

Figure 11 is a geospatial view of the data collected by K10. As the image shows, we collected recon data from six zones at BPLF, with the majority of the data taken at a central basin area. In total, we performed 52 hours of robotic recon, including recon traverse planning, robot operations, and science data analysis. K10 operated for 40 hours, of which 15 hours was productive time (time acquiring recon data) [9].

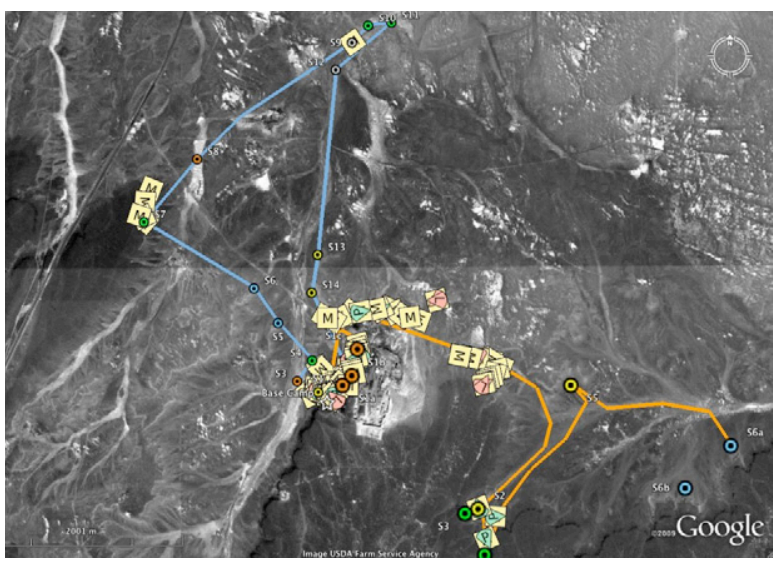

Figure 11. K10 collected recon data from six zones at BPLF. The two "pre-recon" crew traverses are shown in blue (W1) and orange (N2).

\section{POST-RECON PLANNING}

After completion of the robotic recon mission simulation, the science team reviewed the collected data. They then revised the pre-recon traverse plans using the data to reevaluate target science merit, to add/delete stations, to adjust station locations and to modify crew tasks. The resulting post-recon crew traverses were designated $\mathrm{W} 2$ and $\mathrm{N} 2$.

For example, based on recon data, the West science team decided to combine the objectives for W1 Station 2 and W1 Station 4 (Figure 12). The location of the new station, W2 Station 2, is coincident with the location of W1 Station 4.

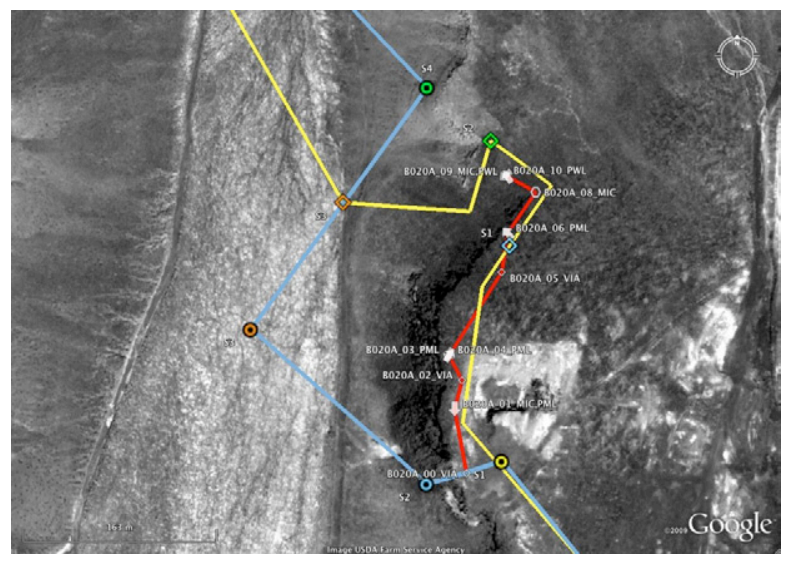

Figure 12. Portions of traverses W1 (blue) and W2 (yellow). K10 recon plan \#B020A is shown in red.

The rationale for this change was as follows:

- Recon indicated that descent from the lava flow surface is better done to the north. 
- The geologic relationships at this location are high priority and all objectives originally tasked for W1 Stations 2 and 4 could be done at a single station.

- A single station allows a reduction in EVA activities and time, which makes it easier to keep within the overall traverse time constraints.

- Removal of W1 Station 2 saves time in W2.

- Consolidation of stations combines a high priority site with a low priority site, which enables multiple objectives to be addressed at a single location.

Similarly, at other targets, the science team made adjustments to more efficiently utilize crew EVA time. Surface features and operational issues that were not detectable from satellite imagery, but that were observable in the recon data, influenced the replanning.

\section{CREW MISSION SIMULATION}

We conducted the final phase of the experiment from 29 August to 3 September 2009 as part of the 2009 D-RATS field test at BPLF. During D-RATS, two crews each performed two single-day traverses (one pre-recon and one post-recon) using the "Lunar Electric Rover" (LER) and simulated EVA suits. Each crew consisted of an astronaut and a field geologist.

The LER (Figure 13) is a prototype pressurized crew rover that is intended to improve human safety and performance in planetary exploration [7]. The LER is slightly larger than the Apollo Lunar Roving Vehicle and provides a pressurized shirt-sleeve environment, along with two "suit ports" for rapid egress and ingress. The LER is equipped with numerous cameras, which provide multiple video channels to ground control. A mast-mounted GigaPan camera can be remotely operated to acquire high-resolution panoramas.

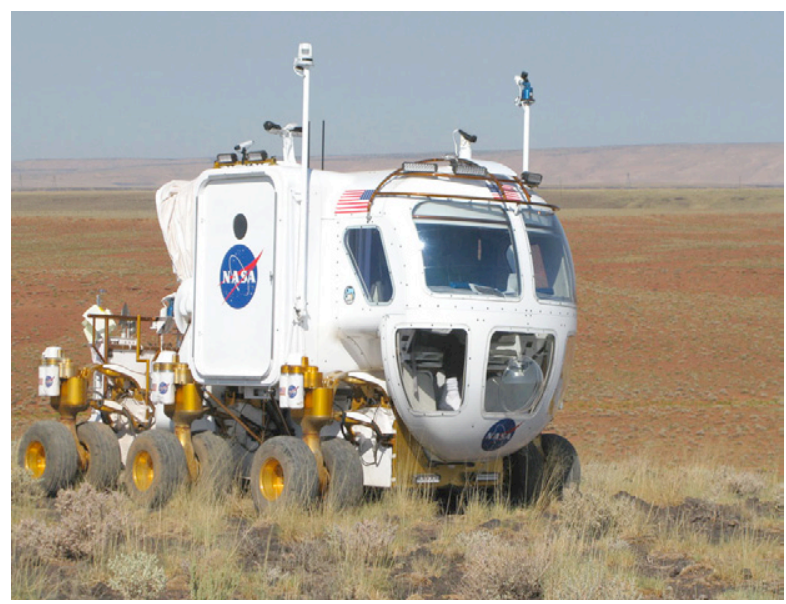

Figure 13. The Lunar Electric Rover at BPLF.

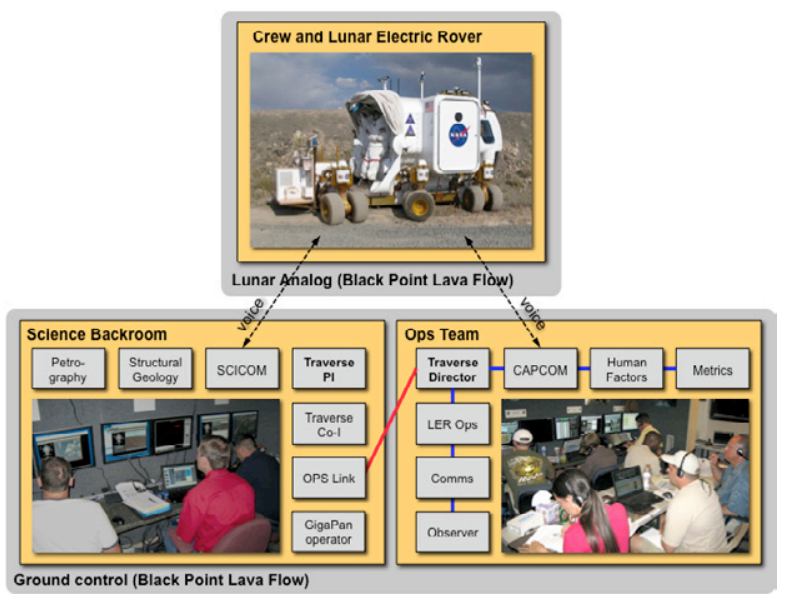

Figure 14. Prototype ground control for lunar crew mission with the LER.

A ground control team (Figure 14) remotely supported the crew throughout the traverses. In this ground control, a "Science Backroom" provided realtime, interactive support to the crew via a voice loop. This backroom operates in a manner similar to the "Science Backroom" used during Apollo, but includes a "SCICOM" operator, who is able to communicate directly with the crew.

The ground control also included an "ops team", which is a simplified flight control team. The ops team performed real-time tactical support, with an emphasis on providing guidance and tracking to a nominal timeline for each traverse plan. As with human flight missions, this team includes a "CAPCOM" operator, who is the designated ops person who communicates with the crew (in coordination with "SCICOM").

Numerous factors impact the performance of field geology with a pressurized rover and EVA suits. These include, but are not limited to:

- crew training, experience, and skill (vehicle, suits, and ops protocols)

- ground control training, experience and skill

- group dynamics (teamwork, communication, etc.)

- trafficability (topography, soil conditions, etc.)

- communications (coverage, bandwidth, etc.)

- vehicle problems (mechanical, avionics, etc.)

- environmental conditions (weather, temperature, illumination, etc.)

- site knowledge (prior visits, reports, etc.)

Each of these factors can be difficult to control during a field test, especially if test time is limited, or if it is impractical to conduct a significant number of trials. 
For the purposes of this experiment, therefore, we chose to control a single variable: use of recon data. Specifically, we ordered traverses such that each crew performed a pre-recon traverse followed by a postrecon traverse. Thus, the first crew performed $\mathrm{N} 1$ then W2; the second crew performed W1 then N2.

For the pre-recon traverses (N1 and W1), we provided the crew and the science backroom with briefing books that contained only traverse maps and satellite images. For the post-recon traverses (N2 and W2), we provided briefing books that also contained images acquired by robotic recon. In addition, during execution of N2 and W2, we gave the science backroom interactive access to all the recon data using the K10 "Ground Data System".

As the crews carried out the traverses, we logged task times, assessed the quality of data, and noted anomalies and potentially biasing events (e.g., inadvertent use of recon data during pre-recon traverses). We also had field observers follow crew and collect ground truth at each target.

\section{PRELIMINARY RESULTS}

We have not yet completed analyzing all the data collected during the robotic recon experiment. However, several important points are already apparent. Most importantly, it is clear that comparing crew traverse plans developed with, and without robotic recon provides significant insight into the benefit of surfacelevel data. However, a quantitative comparison of the executed crew traverses themselves is difficult, given the number of uncontrolled and highly variable factors.

\section{$\underline{\text { Robotic Recon Mission Simulation }}$}

During the robotic recon mission simulation, we monitored robot telemetry and computed performance metrics in real-time. These metrics provide insight into the efficiency of ground control. For example, to maximize data acquisition, the science operations team tried to minimize robot idle time. Thus, robot idle time is indicative of traverse planning efficiency. On average, the science team was able to generate new plans with 31 minutes robot idle time.

We also monitored whether a traverse plan was successfully completed and robot execution time. Over the course of the mission simulation, a total of 37 robot traverse plans were executed. Of these, 17 plans were partially completed, and 20 were fully completed. The large number of partially complete plans reflects: (1) robot performance limitations (i.e., inability to negotiatte some parts of the terrain); and (2) the operations approach we used, which allowed plans to be interrupted (and replanned) based on real-time data.

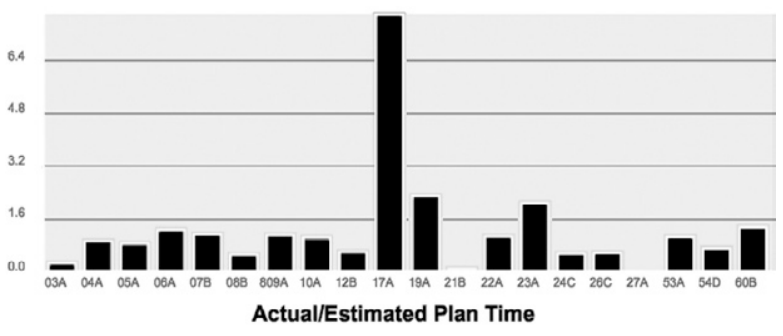

Figure 15. Ratio of actual plan time to estimated plan time. This ratio equals 1.0 for a plan executed in exactly the expected time.

Figure 15 shows the ratio of actual time on plan to estimated time on plan for the robot traverse plans that went to completion. Of the 20 plans that were completed, 16 plans were completed within $10 \%$ of the allocated time.

For lunar recon operations, minimizing all human interaction time may not translate to more efficient recon operations. In fact, it may often be more time and resource efficient to teleoperate the robot in difficult terrain than to operate autonomously. Thus, our objective was to minimize the time spent on unplanned human intervention.

We measured the "Mean Time to Intervene" (MTTI) [10] as the average time humans spent handling anomalies that interrupted robot activity. We also computed the "Mean Time Between Interventions" (MTBI) [10] as the average time between unplanned interventions. Small MTTI and large MTBI indicate good human-robot performance. The average MTTI for the recon mission was 5.6 minutes, ranging from a 1.6 minutes to a of 17.9 minutes. MTBI averaged 24 minutes, ranging from 5.5 minutes to 1 hour [9].

\section{Crew Mission Simulation}

In our crew mission simulation, robotic recon was of major benefit to the West region, because the prerecon traverse (W1) emphasized rapid area coverage and visited several different, widely separated geologic units. From a planning standpoint, this meant that there was a large set of unknowns that recon helped resolve, in terms of both target access (trafficability, route, approach direction) and science content.

In addition, because EVAs were potentially numerous in the West, recon information was essential for prioritizing LER and EVA targets. This was especially true during the $\mathrm{W} 2$ traverse, when the backroom was required to make real-time replanning decisions to accommodate time constraints and changing priorities. In other words, recon enabled the crew and backroom to be more flexible and adaptive during W2, which enabled all the high priority science objectives to be achieved even under difficult field conditions. 
Robotic recon was of less benefit to the North region, primarily because the pre-recon traverse (N1) had a narrower scientific objective, i.e., characterize the BPLF and its contact with the underlying geologic unit. In addition, the recon instruments carried by $\mathrm{K} 10$ had limited capability to address this objective. If $\mathrm{K} 10$ had been equipped with additional instruments (e.g., spectrometers), recon could have focused on identifying candidate targets for sampling.

Consequently, the N1 traverse had fewer scientific uncertainties that could be resolved by recon than the W1 traverses. As a direct result, northern recon focused on reducing operational unknowns: verifying that planned routes were trafficable, identifying and improving precise locations for LER stops, etc.

After all the traverses were complete, we interviewed the crew and asked what recon information would be the most useful to have on-board the LER. Their responses fell into two categories: (1) data to improve situational awareness, such as images of navigation and approach landmarks; and (2) guidelines for operations (e.g., surface roughness map) to help driving and EVA work (e.g., where and what to sample).

\section{DISCUSSION}

\section{Open issues}

The concept of robotic recon is simple: the more information you have, the better you can plan. However, several important questions still need to be answered.

What should be the operations concept for lunar robots? How do the capabilities and operations of robotic rovers need to be changed from current practice ("robot as science instrument") to be used for recon ("robot as scout")? What ground control structure is needed to support scouting activities?

What surface mobility system should be used for scouting? The LER could be used in an unmanned mode. However, this presents a tradeoff: improving understanding of a site prior to crew activity vs. risking damage to the rover before crew arrive. Smaller robots could be used instead, but they may not have sufficient power for long-range operations.

What are the required relationships between crew mobility and recon mobility? In particular, does a recon robot need to have the same performance as a crew rover? Recon may not need to follow the same route as crew (e.g., assessing a descent route into a crater might best be done from an opposing viewpoint), so terrain performance might not need to be the same. Also, there may be significantly greater time for robot missions. Thus, ground speed might not need to be comparable.

How should recon data be processed and presented? The design of tools, displays, and protocols all impact the efficiency of science operations. Given that analysis, decision making, and plan generation can be slow, a key question is: How can we reduce the bottleneck of viewing and analyzing recon data?

How should recon data be logged and georeferenced? Sharing position information among different exploration assets (humans, robots, orbiters) might require absolute positioning, or fixed references. Terrain relative navigation may work for individual visits, but if a sample is identified during recon, a later crew will need to be able to find the exact spot to collect it.

What is the most effective way to coordinate human-robot activity? How can robotic recon data be most rapidly and effectively incorporated into the planning (or replanning) of a crew traverse or an EVA? What scouting data need to be presented to crews in training and during a mission? How and when should this data be conveyed or made available?

\section{Future work}

Given the potential of robotic recon to improve how humans explore the lunar surface, we recommend that further study be performed so as to support the design and development of lunar equipment, training plans, and mission systems. In particular, we recommend that research focus on three objectives.

First, we need to determine how to optimize recon for field exploration. The introduction of robotic activity prior to human fieldwork is a potentially powerful technique for planetary exploration. Several important questions are: How do we adapt robotic recon to specific site and science needs? How much surface science should be performed with the robot vs. done by humans? What instruments are "optimal" for recon?

Second, we need to understand how to optimize science operations during recon. In our work to date, we have found that science analysis and planning is the central bottleneck in recon operations. In contrast to Mars, lunar surface operations can be significantly more interactive and can involve multiple command cycles per day. Thus, finding ways to make science operations rapid and efficient is of critical importance to all future planetary exploration.

Finally, we need to conduct additional field testing to further quantify the impact of robotic scouting on EVA productivity. Our studies indicate that recon can be highly beneficial to crew, improving preparation, situational awareness, and productivity. In order to understand how to best integrate recon into the design of a multi-mission lunar campaign, we need to more thoroughly quantify these benefits. Assessment should focus on empirical measures including performance, efficiency, and reliability as well as qualitative evaluation by experienced field geologists. 


\section{ACKNOWLEDGEMENTS}

We thank Mark Allan, Xavier Bouyssounouse, Lorenzo Flückiger, Linda Kobayashi, Susan Lee, David Lees, Tod Milam, Estrellina Pacis, Eric Park, Liam Pedersen, Trey Smith, Vinh To, and Hans Utz for developing the robotic recon system.

We also thank Mary Sue Bell, Mike Downs, Jim Dumoulin, Marwan Hussein, Frank Jurgens, Tim Kennedy, David Kring, Nina Lanza, Mike Miller, Ann Ollila, Jim Rice, Marc Seibert, Art Snoke, Robert Stewart, Eugene Stoccardo, Jeff Tripp, Mike Wyatt, Aileen Yingst, Kelsey Young and the NASA Lunar Science Institute for supporting the robotic recon mission simulation.

Finally, we sincerely thank the Desert Research and Technology Studies (D-RATS) team and the LER team for their support at Black Point Lava Flow during the crew mission simulation. We would particularly like to acknowledge the efforts of Rob Ambrose, Shaun Azimi, Ernie Bell, Bill Bluethman, Barbara Cohen, Dean Eppler, Cindy Evans, Jake Bleacher, W. Brent Garry, Mike Gernhardt, Jodi Graf, Fred Hörz, Brian Hynek, Joe Kosmo, Gary Lofgren, Chris Looper, Barbara Romig, Andy Thomas, and Daren Welsh.

This work was supported by the NASA "HumanRobotic Systems" project (ESMD Exploration Technology Development Program) and by the NASA Analogs Program (ESMD Directorate Integration Office).

\section{REFERENCES}

1. Fong, T., Bualat, M., et al. 2008. "Field testing of utility robots for lunar surface operations". AIAA2008-7886. In Proceedings of AIAA Space 2008, San Diego, CA.

2. Fong, T., Deans, M., et al. 2008. "Improving lunar surface science with robotic recon". In Proceedings of the Joint Annual Meeting of LEAGICEUM-SRR, Abstract 4049, Cape Canaveral, FL.
3. Deans, M., Fong, T., et al. 2009. "Robotic scouting for human exploration". AIAA-2009-6781. In Proceedings of AIAA Space 2009, Pasadena, CA.

4. Mishkin, A., Limonadi, D., et al. 2006. "Working the Martian night shift. The MER Surface Operations Process". IEEE Robotics and Automation Magazine, Vol. 13(2).

5. Osborn, J. 2006. "The role of the science officer flight controller in the upcoming era of lunar exploration", Mission Operations Directorate, NASA Johnson Space Center, Houston, TX.

6. Romig, B., Kosmo, J., Gernhardt, M., Abercromby, A., 2009. "Desert Research and Technology Studies 2008 Report", In Proceedings of the SAE International Conference on Environmental Systems (ICES), Savannah, GA.

7. Gernhardt, M., Abercromby, A., et al. 2009. "Engineering evaluation of Lunar Electric Rover 1B and Portable Utility Pallet during simulated planetary surface exploration “, EVA Physiology, Systems, \& Performance Project, NASA Johnson Space Center, Houston, TX.

8. Fong, T., Deans, M., et al. 2008. "A Preliminary examination of science backroom roles and activities for robotic lunar surface science". In Proceedings of the NLSI Lunar Science Conference, Abstract 2142, Moffett Field, CA.

9. Schreckenghost, D., Fong, T., et al. 2009. "Measuring robot performance in real-time for NASA robotic recon operations", In Proceedings of the 2009 Performance Metrics for Intelligent Systems Workshop, National Institute of Standards and Technology, Gaithersburg, MD.

10. J. Arnold, "Towards a framework for architecting heterogeneous teams of humans and robots for space exploration", M.S. Thesis, Dept. of Aeronautics and Astronautics, Massachusetts Institute of Technology. 2006. 